\title{
The health service capacity of primary health care in West China: different perspectives of physicians and their patients
}

\author{
Wenjuan Tao ${ }^{1}$, Wenqi Zeng ${ }^{1}$, Ling Yan ${ }^{1}$, Huazhen Yang ${ }^{2}$, Jin Wen ${ }^{1 *}$ and Weimin $\mathrm{Li}^{3}$
}

\begin{abstract}
Background: Many countries, including China, have identified the primary health care system as a reform priority. The purpose of this study is to compare the perceived service capacity of primary care from the perspectives of physicians and their patients in Sichuan province of China.

Methods: A cross-sectional survey was conducted through Quality and Costs of Primary Care (QUALICOPC) questionnaires. A representative sample of 319 primary care physicians and 641 patients in 48 primary healthcare settings were recruited to take part in the study.

Results: Physicians perceived equity of care the best, while quality of care was rated the highest from the perspective of patients. They both regarded coordination as the weakest dimension of primary care service capacity.

Conclusions: Although primary health care reform may have been effective in helping patients acquire better primary care services, our results suggest that coordination is still perceived to be problematic for both physicians and patients. Improving the coordination of care has to be one of the main goals in the future primary care reforms in China.
\end{abstract}

Keywords: Health service capacity, Primary care, QUALICOPC

\section{Background}

Many countries have identified the primary health care system as a priority for reform [1-3]. Primary care (PC) was identified by the Alma-Ata declaration as the foundation for integrating all health and social services to improve health outcomes, and the key to sustainable, accessible, and equitable health systems [4]. In China, a new health care reform was initiated in 2009, focusing on primary care (e.g., instituting universal health insurance coverage, a basic public health service program, and a national essential drug system) [5]. In 2014, the government implemented a hierarchical medical system (involving primary diagnoses at primary health care institutions and two-way referrals among different levels of hospitals), to strengthen the service capabilities of

\footnotetext{
* Correspondence: huaxiwenjin@163.com

${ }^{1}$ Institute of Hospital Management, West China Hospital, Sichuan University,

Guo Xue Xiang 37, 610041 Chengdu, People's Republic of China

Full list of author information is available at the end of the article
}

primary health care and increase reliance upon primary health care services [6].

Evidence from previous studies using administrative data has indicated a significant impact on primary care services since the 2009 reform, e.g., minimum subsidies per capita for basic public health service tripled from 2009 to 2016 [7], and an overall reduction in average prescription costs for patients [8]. However, the government's efforts were not completely successful. People still seek care at relatively high-level hospitals for treatment, which leads to overcrowding in large hospitals and high healthcare expenditures $[9,10]$. Therefore, there is a pressing need to comprehensively evaluate the impact of the 2009 reform on primary health care services in China.

The strength of a country's primary care system depends on multiple dimensions of primary care impacts in the context of its health care system [11]. Previous Chinese studies mainly evaluated the primary care 
service on one dimension, such as equity [12], satisfaction [13] and continuity [14]. Some comprehensive primary care studies have been performed, for example, using surveys such as the Primary Care Assessment Tool (PCAT) [15, 16]. However, these assessments mainly focused on the perceptions of patients, and there is limited research exploring other perspectives, especially primary care physicians. Based on stakeholder theory, evaluating primary care should consider the perspectives of: patients (service users), health care professionals (service providers), and administrators (managers) [17, 18]. It is important to survey primary care physicians because they are the main providers of care in this reform [19]. Furthermore, evaluating primary care from multiple levels better reveals the relationships between the different levels of and provides insight into various stakeholder viewpoints.

The Quality and Costs of Primary Care (QUALICOPC) is an international study of primary care systems designed to understand how patients perceive the quality of primary care, how providers provide services, and overall health outcomes of primary care in 34 countries worldwide [20]. QUALICOPC questionnaires are developed to make a comprehensive analysis of primary care (PC). Evaluation of the service capacity of the PC system includes three levels: structural level (governance, economic conditions, and workforce development), process level (access, continuity of care, coordination of care, and comprehensiveness of care) and outcome level (quality of care, efficiency of care, and equity in health) $[18,19]$.

The aim of our study was to comprehensively evaluate the primary health care service capacity in China by investigating primary care physicians' and their patients' perceptions using the European QUALICOPC protocol. We compared and analyzed similarities and differences between the perspectives of physicians and their patients. Our goal is to contribute evidence for the improvement of Chinese health policy.

\section{Methods}

\section{Setting and sampling}

A cross-sectional survey was conducted in Sichuan province, which is located in the west of China. Sichuan province has a population of 82.62 million and the largest number of primary care institutions of all administrative divisions in China [21]. Multi-stage random cluster sampling was used to make the sample representative. We divided a total of 21 cities in Sichuan province into three levels according to the per capita Gross Regional Product (GRP). Two cities were randomly selected from each level: high-GRP areas (Chengdu, Deyang), middle-GRP areas (Neijiang, Suining) and low-GRP areas (Guangyuan, Aba). Within each city, we randomly selected four Community health centers ( $\mathrm{CHCs}$ ) from urban areas and four township hospitals from rural areas. The $\mathrm{CHCs}$ and township hospitals are the main primary care service providers in China [22]. Finally, no less than 6 physicians and 12 patients were randomly selected from each practice. This study differed from the original QUALICOPC protocol, which surveyed nine consecutive patients for each general practitioner [18]. According to national statistics, in 2016, the average daily number of visits per primary care institution was 9.8 in Sichuan province and 12.9 in China [23]. There were often far less than nine patients for each physician per day, especially in township hospitals. Given this concern, two to three patients per primary physician were recruited in the study.

\section{Survey instrument}

In the QUALICOPC study framework, there are questionnaires for PC physicians (FPS = the Family Physician Survey), their patients (PES=Patient Experiences Survey and PVS=Patient Values Survey) and fieldworkers (PS=Practice Survey) [18]. The original questionnaires were published elsewhere [19]. We adopted the FPS and PES to analyze physician and patient perceptions of the PC service capacity, and the PS to describe primary care settings. The original questionnaires were translated from English to Chinese through a formal forward-back translation process. We performed a cross-cultural adaptation of QUALICOPC questionnaire for use in China, and added some questions about the background of the primary care providers and patients (e.g., the type of medical insurance). Some questions were excluded, either because their content did not apply to China (e.g., "after a patient has been discharged, how long does it usually take to receive a discharge report from the hospital most frequented by your patients," because patients usually receive a discharge report at the time of their discharge in China), or because too few variations were observed in the participants' answers (e.g., place of mother's birth). After translation and adaptation, we conducted a pilot test to validate and revise the questionnaires, making them more applicable and easier to understand. The process of questionnaire adaptation and fieldwork strategy is illustrated in Fig. 1.

\section{Data collection}

Between October 2017 and February 2018, we implemented the survey for primary care physicians and their patients based on the QUALICOPC study protocol $[18,24]$. A total of 319 primary care physicians agreed to participate in the study and completed the FPS. The response rate was $96.4 \%(n=319 / 331)$. For the PES, trained field workers invited patients who had a face-to-face consultation with physicians and who were over the age of 18 years and able to speak and read Chinese. Data were collected at the end of the consultation 




with the physician. Six hundred forty-one patients agreed and completed the patient experience questionnaire, giving a response rate of $91.4 \%(n=641 / 701)$. This study protocol was approved by the Institutional Review Board (IRB) of West China Hospital in Sichuan University. The surveys were carried out anonymously.

\section{Statistical analysis}

To compare the health service capacity of primary care, we developed capacity indicators $(\mathrm{CI})$ for six PC dimensions: "Accessibility" (ACCS), "Continuity" (CONT), "Comprehensiveness" (COMP), "Coordination" (COOR), "Quality of care" (QUAL), "Equity" (EQ). Each dimension was measured with a set of nominal questions (variables) developed by the QUALICOPC project [11]. The actual questions we used to measure different dimensions are provided in Additional file 1. According to Oleszczyk and Krzton-Krolewiecka's calculation method, all variables were evaluated by a scale ranging from -1 (extremely negative) to +1 (extremely positive). The capacity indicator for each dimension was calculated as an arithmetic mean $(\mu)$ of variables. A detailed description of the variable values and data analysis is available in QUALCOPC studies from Poland [17, 25].

Data were entered through the EpiData Software with double entry and validation. Missing data was minimal and was replaced with Mean Imputation (MI). Characteristics of participants were summarized using descriptive statistics. Categorical variables are expressed as proportions. Continuous variables are expressed as means and standard deviations. All analyses were conducted using the Statistical Package for Social Sciences (SPSS) Version 23.0.

\section{Results}

\section{Characteristics of respondents}

Our dataset contained a total 319 PC physician and 641 patient surveys. They were from 48 primary care practices in different level of GDP areas. In the physician sample, the proportion of male and female doctors was similar. The mean age of the participants was 39.4 (SD $=12.1$ ), and their mean years of experience in PC was 17.21 ( $\mathrm{SD}=12.26)$. Nearly half of the physicians worked in general practice, the rest in traditional Chinese medicine and other specialties. In the patient sample, the mean age of respondents was $53.1(\mathrm{SD}=11.3)$. Only $11.2 \%$ had a college education or higher. More than half reported their health status was fair. The detailed socio-demographic characteristics of the study participants is presented in Table 1.

As for the general characteristics of the practices, the 48 primary care practices were located almost equally between urban and rural areas. Few primary care institutions indicated clear information on opening hours (25.0\%) and how to get out-of-hours care (20.8\%). In practices that are not on the ground floor, 19 practices (39.58\%) didn't provide an elevator. Almost all (95.8\%) practices had no handicap adjusted toilet. Only $31.2 \%$ of practices were easily accessible for patients using a wheelchair or a stroller.

\section{Perceived capacity of primary health care service}

The mean values and standard deviations of each dimension of the capacity index are presented in Table 2. From the perspective of primary care physicians, the dimensions ranging from best to worst according to the scores were equity (0.63), accessibility (0.55), quality (0.47), 
Table 1 Socio-demographic characteristics of study participants by GRP area

\begin{tabular}{|c|c|c|c|c|}
\hline Characteristic & Total n (\%) & High-GRP areas $\mathrm{n}(\%)$ & Middle-GRP areas $n(\%)$ & Low-GRP areas $\mathrm{n}(\%)$ \\
\hline \multicolumn{5}{|l|}{ Physicians } \\
\hline Total & $319(100)$ & $107(33.5)$ & $115(36.1)$ & $97(30.4)$ \\
\hline \multicolumn{5}{|l|}{ Gender } \\
\hline Male & $159(49.8)$ & $49(45.8)$ & 57 (49.6) & $53(54.6)$ \\
\hline Female & $160(50.2)$ & $58(54.2)$ & $58(50.4)$ & $44(45.4)$ \\
\hline \multicolumn{5}{|l|}{ Age (years) } \\
\hline$<30$ & $75(23.5)$ & $26(24.3)$ & $32(27.8)$ & $17(17.5)$ \\
\hline $30-$ & $96(30.1)$ & $36(33.6)$ & $32(27.8)$ & $28(28.9)$ \\
\hline $40-$ & 89 (27.9) & $27(25.2)$ & $34(29.6)$ & $28(28.9)$ \\
\hline$\geq 50$ & $59(18.5)$ & $18(16.8)$ & $17(14.8)$ & $24(24.7)$ \\
\hline \multicolumn{5}{|l|}{ Education } \\
\hline High school or below & $3(0.9)$ & $1(0.9)$ & $1(0.9)$ & $1(1.0)$ \\
\hline Junior college & $185(58.0)$ & $55(51.4)$ & $74(64.3)$ & $56(57.7)$ \\
\hline College & $131(41.1)$ & $51(47.7)$ & $40(34.8)$ & $40(41.2)$ \\
\hline \multicolumn{5}{|l|}{ Practice area } \\
\hline Urban & $136(42.6)$ & $51(47.7)$ & $51(44.3)$ & $34(35.1)$ \\
\hline Rural & $183(57.4)$ & $56(52.3)$ & $64(55.7)$ & $63(64.9)$ \\
\hline \multicolumn{5}{|l|}{ Experience in PC (years) } \\
\hline$<5$ & $55(17.2)$ & $17(15.9)$ & $24(20.9)$ & $14(14.4)$ \\
\hline $5-$ & 89 (27.9) & $35(32.7)$ & $34(29.6)$ & $20(20.6)$ \\
\hline $15-$ & $91(28.5)$ & $31(29.0)$ & $34(29.6)$ & $26(26.8)$ \\
\hline $25-$ & $84(26.3)$ & $24(22.4)$ & $23(20.0)$ & $37(38.1)$ \\
\hline \multicolumn{5}{|l|}{ Specialization } \\
\hline General practice & $157(49.2)$ & $55(51.4)$ & $52(45.2)$ & $50(51.5)$ \\
\hline Traditional Chinese medicine & $112(35.1)$ & $35(32.7)$ & $46(40.0)$ & $31(32.0)$ \\
\hline Specialist and other & $50(15.7)$ & $17(15.9)$ & $17(14.8)$ & $16(16.5)$ \\
\hline \multicolumn{5}{|l|}{ Patients } \\
\hline Total & $641(100)$ & $233(36.3)$ & $192(30.0)$ & $216(33.7)$ \\
\hline \multicolumn{5}{|l|}{ Gender } \\
\hline Male & $301(47.0)$ & $105(45.1)$ & $98(51.0)$ & $98(45.4)$ \\
\hline Female & $340(53.0)$ & $128(54.9)$ & $94(49.0)$ & $118(54.6)$ \\
\hline \multicolumn{5}{|l|}{ Age (years) } \\
\hline $18-$ & $56(8.7)$ & $18(7.7)$ & $18(9.4)$ & $20(9.3)$ \\
\hline $40-$ & $211(32.9)$ & $82(35.2)$ & $49(25.5)$ & $80(37.0)$ \\
\hline $50-$ & $165(25.7)$ & $65(27.9)$ & $50(26.0)$ & $50(23.1)$ \\
\hline$\geq 60$ & $209(32.6)$ & $68(29.2)$ & $75(39.1)$ & $66(30.6)$ \\
\hline \multicolumn{5}{|l|}{ Education } \\
\hline Primary school or illiteracy & $190(29.6)$ & $59(25.3)$ & $61(31.8)$ & $70(32.4)$ \\
\hline Middle school & $190(29.6)$ & $53(22.7)$ & $71(37.0)$ & $66(30.6)$ \\
\hline High school & $189(29.5)$ & $63(27.0)$ & $52(27.1)$ & $74(34.3)$ \\
\hline College or higher education & $72(11.2)$ & $58(24.9)$ & $8(4.2)$ & $6(2.8)$ \\
\hline \multicolumn{5}{|l|}{ Employment status } \\
\hline Employed & $288(44.9)$ & $115(49.4)$ & $90(46.9)$ & $83(38.4)$ \\
\hline Self-employed or family business & $223(34.8)$ & $60(25.8)$ & $67(34.9)$ & $96(44.4)$ \\
\hline
\end{tabular}


Table 1 Socio-demographic characteristics of study participants by GRP area (Continued)

\begin{tabular}{|c|c|c|c|c|}
\hline Characteristic & Total n (\%) & High-GRP areas n (\%) & Middle-GRP areas $n(\%)$ & Low-GRP areas n (\%) \\
\hline Retired/unemployed & $130(20.3)$ & $58(24.9)$ & $35(18.2)$ & $37(17.1)$ \\
\hline \multicolumn{5}{|c|}{ Self-evaluated health status } \\
\hline Very good & $35(5.5)$ & $9(3.9)$ & $13(6.8)$ & $13(6.0)$ \\
\hline Good & $136(21.2)$ & $51(21.9)$ & $44(22.9)$ & $41(19.0)$ \\
\hline Fair & $346(54.0)$ & $127(54.5)$ & 99 (51.6) & $120(55.6)$ \\
\hline Poor & $124(19.3)$ & $46(19.7)$ & $36(18.8)$ & $42(19.4)$ \\
\hline \multicolumn{5}{|c|}{ Declared household income } \\
\hline Below average & $326(50.9)$ & $119(51.1)$ & $100(52.1)$ & $107(49.5)$ \\
\hline Average & $315(49.1)$ & $114(48.9)$ & $92(47.9)$ & $109(50.5)$ \\
\hline Above average & $0(0)$ & $0(0)$ & $0(0)$ & $0(0)$ \\
\hline \multicolumn{5}{|l|}{ Chronic disease } \\
\hline Yes & $428(66.8)$ & $164(70.4)$ & $131(68.2)$ & $133(61.6)$ \\
\hline No & 213 (33.2) & 69 (29.6) & $61(31.8)$ & 83 (38.4) \\
\hline
\end{tabular}

Note: GRP Gross Regional Product, PC Primary Care

continuity (0.40), comprehensiveness (0.34), and coordination (0.01). In the equity dimension (the best), the majority $(87.8 \%)$ of the physicians reported that there were no restrictions when accepting new patients. Almost half of the physicians almost always provided health care to people even if they were not remunerated. In the coordination dimension (the worst), only $25.1 \%$ always or usually received new patients' medical records from the previous doctor. The detailed questions and responses on equity and coordination dimension are given in Additional file 2.

From the patient perspective, the dimensions ranging from best to worst according to the scores were quality (0.73), comprehensiveness (0.53), equity (0.52), continuity (0.31), accessibility (0.20), and coordination (0.17). In the quality dimension (the best), over $90 \%$ of patients felt that their doctors were polite and listened carefully, and the vast majority of patients $(96.4 \%)$ were willing to recommend their doctors to friends or relatives. In the coordination dimension (the worst), only a few patients

Table 2 Capacity indexes of the core dimensions in primary care in China

\begin{tabular}{llllll}
\hline Item & \multicolumn{2}{l}{ Physician } & & & Patient \\
\cline { 2 - 3 } & $\bar{x}$ & SD & & $\bar{x}$ & SD \\
\hline Accessibility & 0.55 & 0.20 & & 0.20 & 0.12 \\
Continuity & 0.40 & 0.16 & & 0.31 & 0.33 \\
Coordination & 0.01 & 0.14 & & 0.17 & 0.27 \\
Comprehensiveness & 0.34 & 0.09 & & 0.53 & 0.26 \\
Quality & 0.47 & 0.37 & & 0.73 & 0.20 \\
Equity & 0.63 & 0.20 & & 0.52 & 0.26 \\
\hline
\end{tabular}

Note: $x$ - mean values, SD- standard deviation

Calculation method: all variables were evaluated by a scale ranging from -1 (extremely negative) to +1 (extremely positive). The capacity indicator for each dimension was calculated as an arithmetic mean $(\mu)$ of variables
(5.5\%) reported that their GP informed the medical specialist about their illness when they were referred, and 5.6\% thought their GP knew the results after treatment by a medical specialist. The detailed questions and responses on the quality and cooperation dimensions are given in Additional file 2.

Figures 2 and 3 present distributions of capacity indexes for the core dimensions in primary care and show the differences in perspectives of physicians and patients about the primary care health service capacity. Physicians reported higher scores than patients in the accessibility, continuity, and equity dimensions. In contrast,

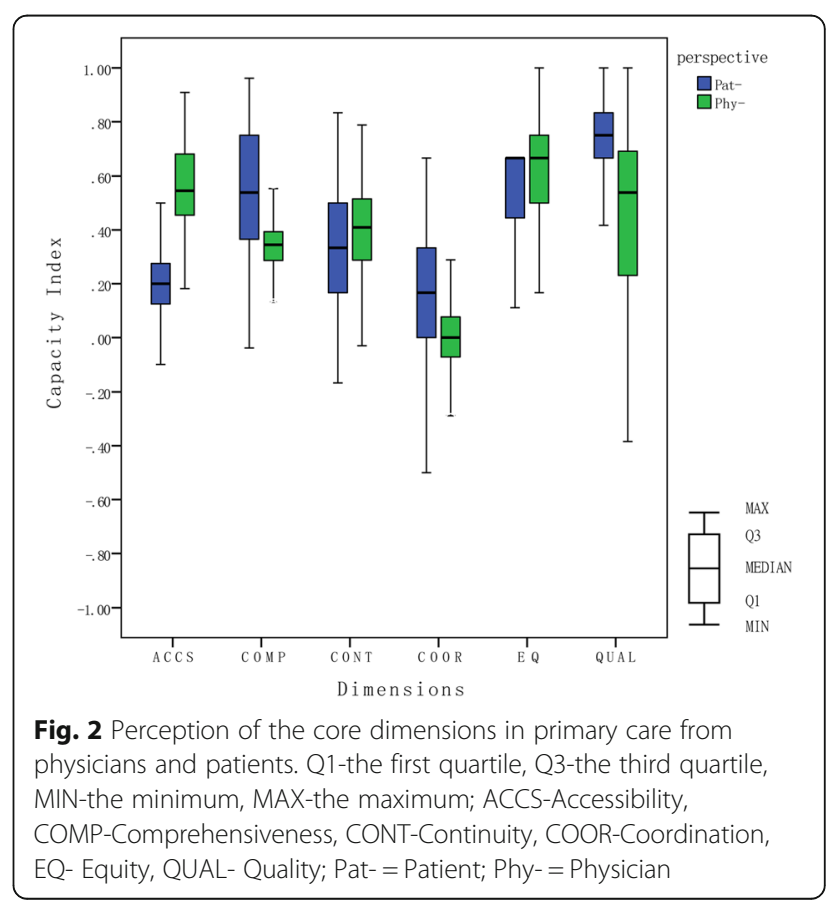






Fig. 3 Differences in the perspectives of physicians and patients

patients reported higher scores in the coordination, comprehensiveness, and quality dimensions than physicians. Both physicians and patients perceived that coordination is the weakest dimension of the primary care service. The largest gap in perceived capacity index between physicians and patients was in the accessibility dimension. $24.8 \%$ of the patients thought the practice was too far away from their living or working places and nearly one-third of patients usually take more $20 \mathrm{~min}$ to travel from their home to the practice. The detailed information of patients on the accessibility dimension are given in Additional file 2.

\section{Discussion}

To the best of our knowledge, this is the first study to measure the primary care service delivery using the QUALICOPC survey in China, and this is also the first study to compare both the perspectives of physicians and patients by QUALICOPC in the world. Our study showed differences and similarities in the core dimensions of the primary care service capacity between physicians and patients in west China.

Firstly, the perception of the best dimension was different among the physicians and the patients. From the point of view of PC physicians, equity of care scored the best. One explanation for such a finding could be that most PC physicians treated their patients fairly, e.g., had no restrictions to accepting new patients, and prescribed the cheapest equivalent medicine to reduce financial obstacles to disadvantaged patients. An alternative explanation for this finding is that the insurance coverage expansion promoted equity in economic access to primary care [26]. In our study, only a few patients (29, $4.5 \%$ ) postponed or abstained from a visit to a physician because of lack of insurance. Previous studies revealed that medical insurance may have played an important role in health equity [27, 28]. Quality of service ranks highest among the dimensions in the perspective of patients. This is inconsistent with a previous study that found the Chinese primary health care system is poor in quality [5]. This difference in findings is probably because the other study focused on outcome measures, while the variables in our study are mainly process measures (e.g., communication, careful treatment).

In addition, physicians had higher perceptions of accessibility, while patients' experiences with accessibility seemed to be relatively worse. In general, accessibility was perceived well in terms of access to services, e.g., making an appointment for a visit and waiting time for consultation in primary care. Our finding that there was a barrier to spatial accessibility of primary care is consistent with literature on this topic [29]. Poor accessibility scores for patients also may relate to the practice characteristics; for example, few primary care institutions outside provided clear information on when open and how to get out-of-hours care.

Lastly, coordination ranked the lowest among the core dimensions from the perspective of both physicians and patients. Similarly, Polish GPs and patients had lower perceptions of coordination [17, 25]. The lack of efficient information flow could be the explanation of worse evaluation of coordination of care. The results showed that medical records were seldomly provided by the previous doctor and results after treatment were often not known when patients transferred. Findings of recent studies indicated that the coordination dimension was more related to the dissemination of information among family physicians or between family physicians and specialists (primary and secondary care) $[25,30]$. Small private practices which may lead to "a culture of individualism" could also impede coordination of care [31]. An alternative interpretation is that the coordination of care is relevant to planners of PC and the opportunities offered by health managers in the local community [32].

In China, poor coordination may be explained by "isolated" and "fragmented" healthcare services, for example, primary healthcare centers and hospitals operate independently and compete for patients [33, 34]. Integrated care has been suggested as one strategy for promoting coordinated healthcare delivery. In 2016, the report on the Deepening Health Reform in China proposed a hierarchical medical system in accordance with a peoplecentered integrated care model for strengthening health care [35]. However, the implementation of this policy has not brought about as many improvements as expected. The referral rate in China was far lower than the general referral rate $(20-30 \%)$ published by the World Health Organization [36]. The previous studies indicated that this may relate to the lack of coordination and continuity between hospitals at the different 
levels [6, 37, 38]. We suggest that policy makers should focus more on the coordination dimension of primary care when enacting heath policy reforms. Sharing medical information (e.g., electronic medical records), shared management (e.g., collaboration skills), and payment stimulus could be the suggestions to promote the coordination [39-41].

Our study has several limitations. Although the questionnaires were designed and validated for an international study and our material allows for international comparisons, the questions were not specifically designed to map the context of China. Thus, for Chinese circumstances, we removed or added some items, and made some adaptations in the original questionnaires. These may lead to a bias in the comparability of our findings with international results. Another limitation is that all information was based on the physician and patient self-reported data. Answers are subjective and could be under- or over-reported, and, therefore, could be inaccurate. Recall bias may also apply. Due to limited clinical knowledge, it is not possible to assess certain aspects of technical quality from the patient's perception. In addition, further research needs to expand the sample size to more primary care settings or regions in China to increase generalizability of findings.

\section{Conclusion}

This study provided an evaluation of the service capacity of primary health care in one province in China using the QUALICOPC protocol. Although primary health care reform may have been effective in helping patients acquire better primary care services, our results suggest that coordination is still perceived to be problematic for both physicians and patients. Improving the coordination of care has to be one of the main goals in the future primary care reforms in China. More efforts are needed to improve the coordinated relationship among primary care physicians and between primary and secondary care physicians. Lessons from primary care reform based on this study can also serve as reminder for other low-income and middle-income countries undertaking similar endeavors in the future. In addition, we have demonstrated that one method of comparing multi-stakeholder's perceptions may help effectively to assess primary care service capacity.

\section{Additional files}

Additional file 1: Questions of GP questionnaire and Patient Experiences questionnaire in the core dimensions. (DOCX $27 \mathrm{~kb}$ )

Additional file 2: Responses of primary care physicians and their patients. (DOCX $38 \mathrm{~kb}$ )

\section{Abbreviations}

CHCs: Community health centers; FPS: the Family Physician Survey: GRP: Gross Regional Product; MI: Mean Imputation; PC: Primary care; PCAT: Primary Care Assessment Tool; PES: Patient Experiences Survey; PS: Practice Survey; PVS: Patient Values Survey; QUALICOPC: Quality and Costs of Primary Care

\section{Acknowledgements}

The authors thank all the teachers, students and the research participants who took part in the data collection. We thank the China Scholarship Council for supporting the first author's visit to the Department of Health Policy and Management at University of California, Los Angeles (CSC Number: 201806240304). We would also like to acknowledge Dr. Gerald F. Kominski and Dr. Zhuyue Li for their assistance in making this a better article.

\section{Funding}

The National Natural Science Foundation of China (Grant No. 71874115) and Science \&Technology Department of Sichuan Province, China (Grant No. 2018KZ0046) financially supported this study. The National Natural Science Foundation of China and Science \&Technology Department of Sichuan Province did not play a role in the design of the study and collection, analysis, and interpretation of data and in writing the manuscript.

\section{Availability of data and materials}

The datasets generated and analyzed during the current study are not publicly available due to restrictions stated in our ethical approvals, but are available from the corresponding author on reasonable request.

\section{Authors' contributions}

JW was involved in the design of this study. WJ-T has written the manuscript that has been critically revised by JW and WM-L. WQ-Z and LY were in charge of the implementation of the data collection in China. WJ-T and HZ-Y analyzed the data and produced all tables and figures. All authors read and approved the final version of the manuscript.

Ethics approval and consent to participate

This study protocol was approved by the Institutional Review Board (IRB) of West China Hospital in Sichuan University on the 25th of September 2017 (covering the study sites: Chengdu, Deyang, Neijiang, Suining, Guangyuan, $A B a)$. It is an anonymous survey for protecting the privacy of participants. The physicians and patients provided their informed oral consent for their participation, any participant who did not wish to participate was given an opportunity to leave before questionnaire survey started. Participants completed the questionnaire by themselves, except those with low level of education and disability with the help of a field worker.

Consent for publication

Not applicable.

Competing interests

The authors declare that they have no competing interests.

\section{Publisher's Note}

Springer Nature remains neutral with regard to jurisdictional claims in published maps and institutional affiliations.

\section{Author details}

${ }^{1}$ Institute of Hospital Management, West China Hospital, Sichuan University, Guo Xue Xiang 37, 610041 Chengdu, People's Republic of China. 'West China School of Public Health, Sichuan University, Chengdu, Sichuan, China. ${ }^{3}$ Department of Respiratory Medicine, West China Hospital of Sichuan University, Chengdu, Sichuan, China.

Received: 18 November 2018 Accepted: 20 February 2019

Published online: 28 February 2019

\section{References}

1. Carter R, Riverin B, Levesque JF, Gariepy G, Quesnel-Vallee A. The impact of primary care reform on health system performance in Canada: a systematic review. BMC Health Serv Res. 2016;16:324. 
2. Lionis C, Papadakis S, Tatsi C, Bertsias A, Duijker G, Mekouris PB, Boerma W, Schafer W. Informing primary care reform in Greece: patient expectations and experiences (the QUALICOPC study). BMC Health Serv Res. 2017;17(1):255.

3. Wexler R, Hefner J, Welker MJ, McAlearney AS. Health care reform: possibilities \&amp; opportunities for primary care. J Fam Pract. 2014;63(6): 298-304.

4. Chokshi DA, Cohen L. Progress in primary care-from Alma-Ata to Astana. JAMA. 2018:320(19):1965-6.

5. Li X, Lu J, Hu S, Cheng K, De Maeseneer J, Meng Q, Mossialos E, Xu DR, Yip W, Zhang $\mathrm{H}$, et al. The primary health-care system in China. Lancet. 2017; 390(10112):2584-94

6. Wang Y, Sun L, Hou J. Hierarchical medical system based on big data and Mobile internet: a new strategic choice in health care. JMIR Med Inform. 2017;5(3):e22.

7. Li L, Fu H. China's health care system reform: Progress and prospects. Int J Health Plann Manag. 2017;32(3):240-53.

8. Yao Q, Liu C, Ferrier JA, Liu Z, Sun J. Urban-rural inequality regarding drug prescriptions in primary care facilities - a pre-post comparison of the National Essential Medicines Scheme of China. Int J Equity Health. 2015;14:58.

9. Liu Y, Zhong L, Yuan S, van de Klundert J. Why patients prefer high-level healthcare facilities: a qualitative study using focus groups in rural and urban China. BMJ Glob Health. 2018;3(5):e854.

10. Cheng JM, Yuan YX, Lu W, Yang L. Primary health care in China: is China's health reform reform for the whole nation? Prim Health Care Res Dev. 2017; 18(4):398-403.

11. Kringos DS, Boerma WG, Hutchinson A, van der Zee J, Groenewegen PP. The breadth of primary care: a systematic literature review of its core dimensions. BMC Health Serv Res. 2010;10:65.

12. Zhong C, Kuang L, Li L, Liang Y, Mei J, Li L. Equity in patient experiences of primary care in community health centers using primary care assessment tool: a comparison of rural-to-urban migrants and urban locals in Guangdong, China. Int J Equity Health. 2018;17(1):51.

13. Li J, Wang $P$, Kong $X$, Liang $H$, Zhang $X$, Shi L. Patient satisfaction between primary care providers and hospitals: a cross-sectional survey in Jilin province, China. Int J Qual Health Care. 2016;28(3):346-54.

14. Liu C, Wu Y, Chi X. Relationship preferences and experience of primary care patients in continuity of care: a case study in Beijing, China. BMC Health Serv Res. 2017:17(1):585.

15. Kuang L, Liang Y, Mei J, Zhao J, Wang Y, Liang H, Shi L. Family practice and the quality of primary care: a study of Chinese patients in Guangdong Province. Fam Pract. 2015;32(5):557-63.

16. Hu R, Liao Y, Du Z, Hao Y, Liang H, Shi L. Types of health care facilities and the quality of primary care: a study of characteristics and experiences of Chinese patients in Guangdong Province, China. BMC Health Serv Res. 2016;16(a):335.

17. Krzton-Krolewiecka A, Oleszczyk M, Schafer W, Boerma WG, Windak A Quality of primary health care in Poland from the perspective of the physicians providing it. BMC Fam Pract. 2016;17(1):151.

18. Schafer WL, Boerma WG, Kringos DS, De Maeseneer J, Gress S, Heinemann S, Rotar-Pavlic D, Seghieri C, Svab I, Van den Berg MJ, et al. QUALICOPC, a multi-country study evaluating quality, costs and equity in primary care. BMC Fam Pract. 2011;12:115.

19. Schafer WL, Boerma WG, Kringos DS, De Ryck E, Gress S, Heinemann S, Murante AM, Rotar-Pavlic D, Schellevis FG, Seghieri C, et al. Measures of quality, costs and equity in primary health care instruments developed to analyse and compare primary care in 35 countries. Qual Prim Care. 2013;21(2):67-79.

20. Premji K, Ryan BL, Hogg WE, Wodchis WP. Patients' perceptions of access to primary care: analysis of the QUALICOPC patient experiences survey. Can Fam Physician. 2018;64(3):212-20.

21. Statistical Bureau Of Sichuan. NBS survey office in Sichuan: Sichuan Statistical Yearbook. Beijing: China Statistics Press; 2017.

22. Yip WC, Hsiao WC, Chen W, Hu S, Ma J, Maynard A. Early appraisal of China's huge and complex health-care reforms. Lancet. 2012;379(9818):833-42.

23. National Health and Family Planning Commission. China health and family planning statistical yearbook 2017. Beijing: Peking Union Medical College Press; 2017.

24. Schafer WL, Boerma WG, Murante AM, Sixma HJ, Schellevis FG, Groenewegen PP. Assessing the potential for improvement of primary care in 34 countries: a cross-sectional survey. Bull World Health Organ. 2015; 93(3):161-8.
25. Oleszczyk M, Krzton-Krolewiecka A, Schafer W, Boerma W, Windak A. Experiences of adult patients using primary care services in Poland - a cross-sectional study in QUALICOPC study framework. BMC Fam Pract. 2017;18(1):93.

26. Junxiang L. YMJX: an evaluation on equity in current primary healthcare reform in China. Asian Bioethics Review. 2015;7(3):277-91.

27. Sun X, Zhang H, Hu X, Gu S, Zhen X, Gu Y, Huang M, Wei J, Dong H. Measurement and analysis of equity in health: a case study conducted in Zhejiang Province, China. Int J Equity Health. 2018;17(1):36.

28. Liu GG, Zhao Z, Cai R, Yamada T, Yamada T. Equity in health care access to: assessing the urban health insurance reform in China. Soc Sci Med. 2002; 55(10):1779-94.

29. Wang X, Yang H, Duan Z, Pan J. Spatial accessibility of primary health care in China: a case study in Sichuan Province. Soc Sci Med. 2018;209:14-24.

30. Cohidon C, Wild P, Senn N. Patient experience in primary care: association with patient, physician and practice characteristics in a fee-for-service system. Swiss Med Wkly. 2018;148:w14601.

31. Pavlic DR, Sever M, Klemenc-Ketis Z, Svab I. Process quality indicators in family medicine: results of an international comparison. BMC Fam Pract. 2015:16:172.

32. Pavlic DR, Sever M, Klemenc-Ketis Z, Svab I, Vainieri M, Seghieri C, Maksuti A. Strength of primary care service delivery: a comparative study of European countries, Australia, New Zealand, and Canada. Prim Health Care Res Dev. 2018:19(3):277-87.

33. Yip W, Hsiao W. Harnessing the privatisation of China's fragmented healthcare delivery. Lancet. 2014:384(9945):805-18

34. Tang W, Wei L, Zhang L. Analysing a Chinese regional integrated healthcare organisation reform failure using a complex adaptive system approach. Int J Integr Care. 2017;17(2):3.

35. Wang X, Sun X, Birch S, Gong F, Valentijn P, Chen L, Zhang Y, Huang Y, Yang H. People-centred integrated care in urban China. Bull World Health Organ. 2018;96(12):843-52.

36. Yu W, Li M, Nong X, Ding T, Ye F, Liu J, Dai Z, Zhang L. Practices and attitudes of doctors and patients to downward referral in Shanghai, China. BMJ Open. 2017:7(4):e12565.

37. Shi G, Zhou B, Cai ZC, Wu T, Li XF, Xu W. Referral by outreach specialist reduces hospitalisation costs of rural patients with digestive tract cancer: a report from medical consortium in China. Rural Remote Health. 2014;14:2317.

38. Xiong X, Zhou S, Huo Z, Luo L. GIS-based method to delimit spheres of influence for a medical consortium: experience of a pediatric medical consortium, shanghai. Int J Health Plann Manag. 2018;1-15. https://doi.org/ 10.1002/hpm.2630

39. Cai M, Liu E, Tao H, Qian Z, Fu QJ, Lin X, Wang M, Xu C, Ni Z. Does a medical consortium influence health outcomes of hospitalized Cancer patients? An integrated care model in Shanxi, China. Int J Integr Care. 2018:18(2):7.

40. Widmark C, Sandahl C, Piuva K, Bergman D. Barriers to collaboration between health care, social services and schools. Int J Integr Care. 2011:11:e124.

41. Qian Y, Hou Z, Wang W, Zhang D, Yan F. Integrated care reform in urban China: a qualitative study on design, supporting environment and implementation. Int J Equity Health. 2017;16(1):185.

\section{Ready to submit your research? Choose BMC and benefit from:}

- fast, convenient online submission

- thorough peer review by experienced researchers in your field

- rapid publication on acceptance

- support for research data, including large and complex data types

- gold Open Access which fosters wider collaboration and increased citations

- maximum visibility for your research: over $100 \mathrm{M}$ website views per year

At BMC, research is always in progress.

Learn more biomedcentral.com/submission 